INPLASY

PROTOCOL

To cite: Jiang et al. Efficacy and Safety of Acupuncture in Patients with Cancer-Related Fatigue: A Protocol for Systematic Review and MetaAnalysis. Inplasy protocol 202090049. doi: 10.37766/inplasy2020.9.0049

Received: 12 September 2020

Published: 12 September 2020

Corresponding author: Xi Wu

wuxi@cdutcm.edu.cn

Author Affiliation:

Chengdu University of

Traditional Chinese Medicine

Support: NSFC.

Review Stage at time of this submission: Preliminary searches.

Conflicts of interest:

The authors have no conflicts of interest to disclose.

\section{Efficacy and Safety of Acupuncture in Patients with Cancer-Related Fatigue: A Protocol for Systematic Review and Meta-Analysis}

Jiang, TJ1; Zhu, FY2; Tang, LJ3; Liu, ZK4; Wu, X55.

Review question / Objective: The aim of this study was to evaluate the efficacy and safety of acupuncture for patients with cancer-related fatigue.

Condition being studied: According to the survey, in recent years, the cancer is increasing gradually, and the cancerrelated fatigue increase too. The causes of cancer-related fatigue are mostly due to cancer or cancer treatment and other reasons. In the majority of studies, $30 \%$ to $60 \%$ of patients report moderate to severe fatigue during treatment, which in some cases may lead to treatment discontinuation. There has been a systematic evaluation of acupuncture for cancer-related fatigue, but the efficacy is still controversial due to lack of evidence. In recent years, there are many randomized controlled trials that show that acupuncture is effective for cancer-related fatigue, which may change the clinical evidence of acupuncture treatment. Therefore, this study hopes to provide evidence-based evidence for acupuncture treatment of cancer-related fatigue through this systematic evaluation.

INPLASY registration number: This protocol was registered with the International Platform of Registered Systematic Review and Meta-Analysis Protocols (INPLASY) on 12 September 2020 and was last updated on 12 September 2020 (registration number INPLASY202090049).

\section{INTRODUCTION}

Review question / Objective: The aim of this study was to evaluate the efficacy and safety of acupuncture for patients with cancer-related fatigue.
Condition being studied: According to the survey, in recent years, the cancer is increasing gradually, and the cancerrelated fatigue increase too. The causes of 
cancer-related fatigue are mostly due to cancer or cancer treatment and other reasons. In the majority of studies, $30 \%$ to $60 \%$ of patients report moderate to severe fatigue during treatment, which in some cases may lead to treatment discontinuation. There has been a systematic evaluation of acupuncture for cancer-related fatigue, but the efficacy is still controversial due to lack of evidence. In recent years, there are many randomized controlled trials that show that acupuncture is effective for cancer-related fatigue, which may change the clinical evidence of acupuncture treatment. Therefore, this study hopes to provide evidence-based evidence for acupuncture treatment of cancer-related fatigue through this systematic evaluation.

\section{METHODS}

Participant or population: Patients with Cancer-Related Fatigue.

Intervention: Acupuncture (hand acupuncture and electropuncture) or acupuncture combined with other therapies.

\section{Comparator: Non acupuncture.}

Study designs to be included: Randomized controlled clinical trials (RCTs) in the systematic review for evaluation.

Eligibility criteria: Reported in Chinese or English, and meet the "PICOS", will be considered for inclusion in this overview.

Information sources: We will searched the randomized controlled trial literature of acupuncture treatment for CRF in four English and four Chinese databases, including PubMed, MEDLINE, Web of Science, Cochrane Central Register of Controlled Trials (Central), China National Knowledge Infrastructure (CNKI), China Biomedical Literature Database(CBM), China Science Journal Database (VIP) and Wanfang Database.
Main outcome(s): The Cancer-related fatigue scale score.

Additional outcome(s): TCM Syndrome Integral Scale ; adverse event.

Quality assessment / Risk of bias analysis: The "deviation risk" tool in Cochrane Handbook v.5.1.0 is used to assess the deviation risk of each article in the literature. This assessment includes sequence generation, allocation sequence hiding, the blindness of participants, personnel, and outcome evaluators, incomplete outcome data, selective outcome reporting, and other sources of bias. The assessment results are classified into 3 levels, that is, low risk, high risk, and uncertainty risk. If the risk of bias is high in the literature, then we will try to explain and discuss the causes of bias.

Strategy of data synthesis: RevMan software(V.5.3) is used to import data and perform data statistical analysis. For dichotomous data, If $\mathrm{I}^{2}<50 \%$, then the fixed-effect model will be used for data synthesis, if $\mathrm{I}^{2}$ is from $50 \%$ to $75 \%$, then the random-effect model will be conducted for data synthesis, and if $I^{2}>75 \%$, then we will investigate possible reasons from both clinical and methodological perspectives and provide a descriptive analysis or conduct subgroup analysis. On the other hand, for continuous data, if no heterogeneity is detected, the MD or SMD will be used to measure the therapeutic effect of $95 \% \mathrm{CIs}$, and if significant heterogeneity is found, then the randomeffect model will be used instead.

Subgroup analysis: In case the included studies have high heterogeneity, STATA software will be used to explore the potential sources of heterogeneity, according to different combinations of acupuncture and other therapies, different course time, or different outcome indicators.

Sensibility analysis: A sensitivity analysis is conducted to assess the stability of results. It is conducted from different aspects including sample size, method quality, and 
data missing to evaluate its impact on the study. The meta-analysis will be reused and inferior quality research will be excluded.

Language: English and Chinese.

Country(ies) involved: China.

Keywords: Systematic review; metaanalysis; Cancer; fatigue; Acupuncture; Acupuncture Therapy; Humans.

Contributions of each author:

Author 1 - Tai Jun Jiang.

Author 2 - Feng Ya Zhu.

Author 3 - Li Jie Tang.

Author 4 - Zheng Kang Liu.

Author 5 - Xi Wu. 